\title{
Investigación de Almidones Termoplásticos, Precursores de Productos Biodegradables
}

\author{
Héctor S. Villada ${ }^{1 *}$, Harold A. Acosta ${ }^{2}$ y Reinaldo J. Velasco ${ }^{1}$ \\ (1) Universidad del Cauca, Facultad de Ciencias Agropecuarias, Departamento de Agroindustria, \\ Calle 5 No. 4-70, Popayán-Colombia (e-mail: hsamuelv@yahoo.com) \\ (2) Universidad del Valle, Facultad de Ingeniería, Departamento de Ingeniería de Alimentos, \\ Calle 13 No. 100-00, Apdo. Aéreo 25360 Cali-Colombia
}

*autor a quien debe ser dirigida la correspondencia

\begin{abstract}
Resumen
En este trabajo se realizo una revisión enfocada al entendimiento de los conceptos detrás del término Almidón Termoplástico-TPS, para facilitar el desarrollo de productos biodegradables. La revisión de la literatura que abarca el presente trabajo se agrupó en seis categorías: fuentes, características y usos de los almidones; fusión y mecanismos de transformación; verificación del proceso de plastificación y propiedades; modificaciones químicas y bioquímicas; envejecimiento y biodegradación; y mezclas para el desarrollo de nuevos materiales. Esta categorización permite comprender los avances en este campo en los últimos quince años, así como los principales retos para el futuro, siendo los más relevantes disminuir la sensibilidad a la humedad y retardar la retrogradación de la matriz termoplástica.
\end{abstract}

Palabras claves: almidón termoplástico, plastificación, precursores, biodegradable

\section{Research on Thermoplastic Starches, Biodegradable Products Precursors}

\begin{abstract}
This paper presents a review focused on the understanding of the concepts behind the term Thermoplastic Starch -TPS to facilitate the development of biodegradable products. The literature review covered in this work is grouped into six categories: sources, characteristics and uses of starches; fusion and mechanisms of transformation; plasticizing properties and verification process; chemical and biochemical changes; aging and biodegradation; and mixtures for the development of new materials. This categorization allows understanding the advances in this field over the past fifteen years, as well as major challenges for the future, being the most important reducing the sensitivity to moisture and retarding the retrogradation of the thermoplastic matrix.
\end{abstract}

Keyword: thermoplastic starch, plasticization, precursors, biodegradable 


\section{INTRODUCCIÓN}

EI TPS es compatible con el medio ambiente, es un material renovable y puede incorporarse al suelo como abono orgánico. La acumulación de materiales plásticos puede contribuir en cierta medida a la contaminación ambiental, aunque la mayoría de los materiales plásticos tradicionales son recalcitrantes (inertes al ataque microbiano), la contaminación que producen es fundamentalmente visual, es por ello que el interés hoy en día se ha dirijo al desarrollo de polímeros biodegradables obtenidos de recursos naturales renovables. En la actualidad hay mucha investigación básica y aplicada sobre el almidón, por ser un polímero natural barato y abundante. El desarrollo y producción de almidón termoplástico biodegradable (Thermoplastic starch, TPS) se considera importante para reducir la cantidad total de desechos plásticos sintéticos en el mundo (Ma y Yu, 2004).

EI TPS es un material que se obtiene por la disrupción (modificación) estructural que se da dentro del gránulo de almidón cuado este es procesado con un bajo contenido de agua y la acción de fuerzas térmicas y mecánicas en presencia de plastificantes que no se evaporan fácilmente durante el procesamiento (Bastioli, 2001). El TPS presenta varios atributos, además de su biodegradabilidad, es un material renovable, flexible y se puede acondicionar muy fácilmente a diferentes procesos de termoplastificación usando equipos estándar utilizados en la fabricación de polímeros sintéticos, tales como inyección por moldeo, extrusión por soplado, moldeo por inyección, moldeo por compresión, extrusión de película plana y radiación por moldeo (Van Soest et al., 1996b; Zhai et al., 2003), aunque se ha reportado la utilización de un nuevo método en la preparación de películas de almidón termoplástico usado en el cubrimiento de alimentos. Este método se llama atomización electrohidrodinámica (EHDA) el cual es un proceso en el que la mezcla de almidón + plastificante es forzada a través de un capilar por la aplicación de una diferencia de potencial, del orden de kilovoltios, entre el capilar y la colección de electrodos, cuyo propósito es controlar el espesor y la uniformidad de la película (Pareta y Edirisinghe, 2006).

Durante los últimos veinte años, varios trabajos de investigación se orientaron hacia la modificación del almidón en la producción de un buen material termoplástico (Avérous y Boquillon, 2004; Bangyekan et al., 2006; Demiate et al., 2002). Algunas mezclas de TPS+Biopolímeros, más o menos exitosas, basadas en almidón termoplástico están siendo comercializadas e impresas por compañías como Mater Bi ${ }^{\circledR}$ (Novamont S.P.A., Italia) en Italia [www.mater-bi.com], Carghill-Down ${ }^{\circledR}$ en Estados Unidos y por otras de España, Alemania, Francia, Japón, Dinamarca y Canadá (Mater Bi, 2007).

Sin embargo, el TPS es un material que generalmente presenta poca estabilidad cuando las condiciones de humedad son muy altas (Avérous y Frigant, 2001; Avérous y Boquillon, 2004; Avérous et al., 2003). Uno de los problemas con el uso del TPS en bioplásticos es su naturaleza frágil, causada relativamente por su baja temperatura de transición vítrea $\mathrm{Tg}$ y la falta de relajación de la sub-Tg debido al encadenamiento molecular del almidón (Kenshi et al., 1999; Shogren et al., 1993). Adicionalmente, la migración eventual de los plastificantes al ambiente aumenta la fragilidad del material (De Graaf et el., 2003). La fragilidad es un problema de estabilidad estructural que se incrementa con el paso del tiempo debido a la disminución del volumen libre y la retrogradación del almidón (Kuakoon et al., 2003). Para aumentar la flexibilidad del TPS, se han utilizado una gran variedad de plastificantes como azúcar, polialcoholes, aminoácidos, lípidos, sorbatos y fosfatos (De Graaf et el., 2003; Fama et al., 2005; Kuakoon et al., 2003; Nashed et al., 2003; Parra et al., 2004; Petersson y Standing, 2005). Algunos estudios sostienen que los compuestos nitrogenados previenen la retrogradación en los geles de almidón más tiempo que los otros aditivos, aumentando la estabilidad del gel (Ma y Yu, 2004; Shogren et al., 1993). Sin embargo, la mayoría de los compuestos nitrogenados se encuentran en estado sólido y se funden a altas temperaturas dando poca flexibilidad (Avérous y Frigant, 2001). Otro problema en el desarrollo de TPS es la presencia de un alto contenido de amilosa por que disminuye la flexibilidad en comparación con los TPS hechos a partir de altos contenido de amilopectina (Van Soest y Essers, 1997). Además, durante el almacenamiento los TPS hechos a partir de almidones nativos experimentan cambios estructurales, presentándose una mayor fragilidad o rigidez dependiendo del contenido de plastificante (Van Soest et al., 1996a). 
La Fig. 1, muestra las tres formas de presentación del almidón termoplástico: TPS en pelet, TPS en espuma y TPS en película, obtenidas por extrusión o moldeo por compresión, que por lo general son procesos de etapas múltiples (Werner y Strobel, 1991).

Las formulaciones varían entre el 50-90 \% de almidón y entre el 50-10\% de aditivos plastificantes. La elaboración del TPS puede manipularse de acuerdo con las condiciones mecánicas y térmicas del proceso de termo-plastificación (Van Soest y Essers, 1997).

Las propiedades reológicas del compuesto final están determinadas en parte por las modificaciones que se le realicen al almidón. Estas condiciones dependen del grado de depolimerización de las cadenas del gránulo del almidón al interior de la matriz termoplástica e influyen en las características del TPS (Werner y Strobel, 1991).

\section{ÁREAS DE INVESTIGACIÓN DEL TPS}

Las investigaciones en los últimos tres lustros (1991-2007), se han enfocado hacia una comprensión minuciosa del TPS. La mayoría de estas investigaciones se pueden agrupar en las siguientes categorías, algunas de las cuales han sido propuestas con anterioridad por diferentes autores (Biological Materials, 1998; Mater Bi, 2007; Novamont, 2007):

- Fuentes, características y usos de los almidones.

- Fusión y mecanismos de transformación.

- Verificación del proceso de plastificación y propiedades.

- Modificaciones químicas y bioquímicas.

- Envejecimiento y biodegradación.

- Mezclas para el desarrollo de nuevos materiales.

Los resultados de las investigaciones en cada una de estas categorías se pueden resumir así:

Fuentes, características y usos de los almidones

El almidón es el carbohidrato más abundante en la naturaleza, es sintetizado durante la fotosíntesis y almacenado en los amiloplastos de la células vegetales (Yuryev et al., 1995). El almidón es un gránulo parcialmente cristalino e insoluble en agua a temperatura ambiente. Los gránulos de almidón tienen diferentes tamaños y formas dependiendo de la fuente biológica de donde provengan. La forma puede ser redonda, elíptica, ovalada, lenticular o poligonal (Hoover, 2001). Los gránulos más pequeños corresponden a los almidones del arroz y amaranto con diámetros entre 1 y $5 \mu \mathrm{m}$. Los gránulos de almidón de papa y yuca presentan diámetros entre 15-100 $\mu \mathrm{m}$ (Bello et al., 1998). El diámetro de los gránulos del almidón de maíz varía entre 25-50 $\mu \mathrm{m}$ según la variedad de maíz (Acosta, 1997; Baldwin, 1995; Yuryev et al., 1995). En general, la superficie de los diferentes gránulos del almidón varía según el origen botánico y constituye un interrogante en investigaciones (Lindeboom et al., 2004). Los gránulos de almidón están formados por dos polímeros: amilosa y amilopectina constituido por unidades de glucosa. La amilosa, generalmente el menor componente,

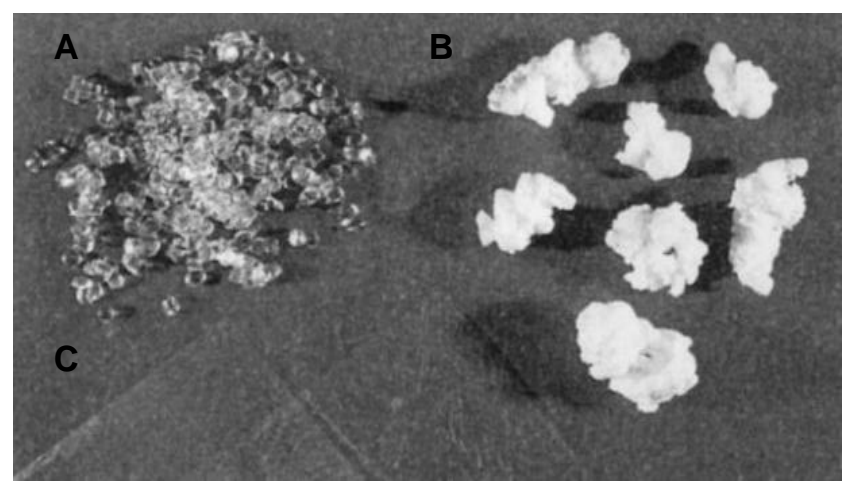

Fig. 1: Almidón termoplástico biodegradable: Pelet (A). Espuma (B). Película (C). 
presenta una estructura lineal constituida por monómeros de glucosa ligados únicamente por enlaces $\alpha-1,4$. La amilosa forma muchos complejos insolubles con un gran número de moléculas que generalmente se precipitan. La amilopectina es generalmente el mayor componente y es altamente ramificada, formada por monómeros de glucosa ligados por enlaces $\alpha-1,4$ y $\alpha-1,6$ (Philippe, 1995). El almidón es el carbohidratos de mayor importancia desde el punto de vista alimenticio después de la glucosa y otros azúcares. Por la amplia gama de posibilidades que ofrecen los almidones en los productos alimenticios modernos, se han convertido desde hace varios años en objeto de amplia investigación científica en el mundo. Los almidones se utilizan en la industria alimentaria para fabricar productos de panadería, postres, pudines, rellenos de tortas, bizcochos, caramelos, gomas dulces, chicles, etc. Los almidones también se usan a escala industrial en papelería, adhesivos, bioempaques, pegantes, floculantes, hidrocoloides, gomas, dextrinas, etc. (Philippe, 1995).

Los almidones nativos o hidrolizados y plastificados, fueron desarrollados bajo diferentes condiciones operativas, porque la producción de materias primas a escala industrial sigue siendo evaluada debido a las diferentes aplicaciones del almidón. Los almidones nativos son aquellos que no han sufrido ninguna modificación y es extraído de fuentes naturales. El almidón esta compuesto de dos polímeros amilosa y amilopectina, la proporción de estos dos polímeros depende de la fuente de origen. La amilosa generalmente es el menor componente, presenta una estructura lineal constituida por monómeros de glucosa ligados únicamente por enlaces $\alpha-1,4$. La amilosa forma muchos complejos insolubles con un gran número de moléculas que generalmente se precipitan. La amilopectina es generalmente el mayor componente y es altamente ramificada, formada por monómeros de glucosa ligados por enlaces $\alpha-1,4$ y $\alpha-1,6$. Los almidones de arveja, trigo, maíz, sorgo, cebada, amaranto, papa, yuca, batata y ñame son extraídos por dos vía (seca y húmeda). En general, la producción de los almidones ha estado variando en el caso de los cereales entre el 65-85 $\%$ y en las raíces y tubérculos entre el 35-15 \% lo cual ha influido de manera significativa en la producción del TPS según la región de influencia del almidón. Los estudios de plastificación también se han relacionado con el tipo de extracción, el tipo de almidón y su organización molecular, demostrándose la incidencia de las variables de operación en el proceso de termoplastificación. (Acosta et al., 2004; Blacido et al., 2005; Fama et al., 2005; Forssell et al., 1997; Mali et al., 2005a; Parra et al., 2004; Shogren et al., 1993; Werner y Strobel, 1991).

\section{Fusión y mecanismos de transformación}

Para que un almidón nativo se pueda procesar y formar un material bioplastico es necesario romper y fundir la estructura semicristalina original (Van Soest et al., 1996a). Se han desarrollado varios métodos para elaborar TPSs. La estabilidad, transformación y las propiedades físicas de los TPSs dependen de la naturaleza de las zonas amorfas y cristalinas presentes en la estructura del granulo. La transformación del almidón granular está influenciada por las condiciones de proceso tales como la temperatura y el contenido de plastificante. El agua y el glicerol son los plastificantes mas comúnmente usados. Durante los diferentes procesos de termoplastificación la influencia del agua y el glicerol en los gránulos del almidón son de gran importancia, dada su acción como lubricante lo cual facilita la movilidad de las cadenas poliméricas. Además, retardan la retrogradación de los productos termoplastificados (Van Soest et al., 1996a).

Las investigaciones mas recientes han apuntado a la comprensión de los procesos de fusión del almidón. Así, se ha dado una descripción teórica de los modelos de fusión según el tipo de cristalinidad A (cereales), B (raíces) y C (leguminosas) en los almidones nativos e hidrolizados, usándose la estadística termodinámica de primer orden (flujo teórico) (Mater Bi, 2007; Myllärinen et al., 2002a; Novamont, 2007). La interacción del almidón nativo con plastificantes no acuosos como glucosa, compuestos nitrogenados, polialcoholes, ácidos grasos, acido cítrico y otros también fue descrita. Estos plastificantes disminuyen la absorción de agua en el almidón lo cual evita que el material se vuelva frágil. Además, la propiedades mecánicas (esfuerzo y elongación) pueden mantener relativamente constates entre los 25-30 días de almacenamiento (Bernal et al., 2005; Fang et al., 2002; Follain et al., 2005; Ma y Yu, 2004; Ma et al., 2007; Myllärinen et al., 2002b; Orts et al., 2007; Shi et al., 2007; Smits et al., 2003; Van Soest et al., 1996c; Wilpiszewska y Spychaj, 2007). 
También se ha descrito la formación de estructuras cristalinas típicas denominadas $V_{H}, V_{A}$ y $E_{H}$ en la matriz termoplástica, las cuales se forman debido a la presencia de hélices simples de amilosa en TPS e igualmente se ha estudiado la influencia de los procesos de recristalización sobre las propiedades mecánicas del almidón termoplástico. Los diferentes procesos de retrogradación se deben principalmente a la absorción de agua al interior de la matriz termoplástica, lo cual permite el reacomodamiento de las cadenas poliméricas gracias a la presencia de grupos $\mathrm{OH}$. La retrogradación afecta entonces las propiedades mecánicas, el esfuerzo aumenta y la elongación disminuye afectando la calidad del bioplástico (Van Soest et al., 1996a; Van Soest y Vliegenthart, 1997; Myllärinen et al., 2002a; Zhang et al., 2007). Los residuos de estructuras cristalinas tipo B, después del proceso de termoplastificación, han sido estudiados por rayos- $\mathrm{X}$, dada su influencia en los proceso de recristalización y su incidencia en las formación de las estructuras $V_{H}, V_{A}$ y $E_{H}$ (García et al., 2000; Myllärinen et al., 2002a). Además, se encontró que estas estructuras están influenciadas por la presencia de agua y plastificante. A menor contenido de agua se forman estructuras cristalinas $V_{A}$ que las estructuras cristalinas $V_{H}$ y las estructuras cristalinas $E_{H}$ se deben a la mezcla de aguaplastificante (García et al., 2000; Van Soest et al., 1996a).

Se analizó el comportamiento reológico durante la extrusión de varios almidones termoplásticos con baja hidratación (Aichholzer y Fritz, 1998; Bindzus et al., 2002a; García et al., 2000; Jiugao et al., 1998), así mismo, se determinó: el efecto del agua, plastificantes y el contenido de amilosa en las características viscoelásticas de los materiales. A medida que aumenta la concentración (agua+plastificante) las propiedades viscoelasticas cambian el esfuerzo disminuye y la elongación aumenta. Además, almidones con alto contenido de amilosa afectan el esfuerzo (aumenta) y la elongación (diminuye) (Bindzus et al., 2002b; García et al., 2000; Nashed et al., 2003). El cambio en el comportamiento reológico dada la presencia de elementos extraños (ceniza) durante la operación de extrusión con tornillo simple o con tornillo doble, se relacionó con el grado de depolimerización del almidón (Aichholzer y Fritz, 1998). Además, el uso de lubricantes (aceites vegetales) en la producción de TPSs ayuda a disminuir la degradación térmica del almidón generada por la depolimerización y mejora las condiciones de proceso (Mali et al., 2002; Onteniente et al., 2000).

Se estudió el comportamiento de la capacidad calorífica específica en sistemas de almidón-aguaplastificante como una función de la temperatura y la composición. El contenido de agua y la temperatura están directamente relacionadas con la capacidad calorífica del sistema, pero la influencia del contenido de glicerol se relaciona con la propiedad térmica según el tipo de almidón que se utilice (Tan et al., 2004).

\section{Verificación del proceso de plastificación y propiedades}

Esta categoría la constituyen estudios que tienen que ver con el seguimiento de la separación de las fases constituidas por los carbohidratos (amilosa/amilopectina) con plastificantes solos o mezclados, en los TPS obtenidos por extrusión o moldeo por compresión (Halley et al., 2003; Myllärinen et al., 2002b; Petersson y Standing, 2005). Además, se incluyen estudios sobre cambios en las propiedades mecánicas y térmicas como función de la composición y de diferentes condiciones de almacenamiento (humedad relativa y tiempo de almacenamiento). Durante el almacenamiento el contenido de plastificante disminuye debido a la migración de este, desde la matriz a la superficie y al medio ambiente, afectando las propiedades mecánicas (el esfuerzo aumenta y la elongación disminuye). Igualmente, las propiedades térmicas cambian aumentan las temperaturas de transición vítrea y fusión (Halley et al., 2003; Zhang y Han, 2006). La permeabilidad a gases $\left(\mathrm{O}_{2}, \mathrm{CO}_{2}\right.$ y vapor de agua) se ha estudiado según el tipo de plastificante usado. La permeabilidad aumenta a medida que la concentración del plastificante aumenta siendo mayor en compuestos hidrófilos que en hidrófobos (Dole et al., 2004; Forssell et al., 2002; Finkenstadt y Willett, 2004; Rhim, 2004; Stepto, 2006).

Durante el almacenamiento, el envejecimiento fue evidente debido a los diferentes procesos de recristalización de las cadenas poliméricas y la difusión de los plastificantes al medio externo en el TPS. Este fenómeno de recristalización (retrogradación) depende de las condiciones ambientales. Lo cual incide en la separación de fases entre el plastificante y el almidón (Gaudin et al., 1999; Tsiapouris y Linke, 2002; Yang et al., 2006; Yuryev et al., 1995). 


\section{Modificaciones químicas y bioquímicas}

En esta categoría se incluyen los estudios que tienen ver con modificaciones químicas y enzimáticas durante el proceso de extrusión (tornillo simple o doble) conocida como extrusión reactiva (Brown y Orlando, 1988) y también los estudios relacionados con la incorporación de fibras (Avérous et al., 2001; Curvelo et al., 2001; Nashed et al., 2003; Xie et al., 2006). Otras investigaciones muestran que la modificación del almidón a través de la esterificación, eterificación y la oxidación antes del proceso de termoplastificación permite lograr un alto grado de sustitución de grupos hidrofilicos -OH por grupos hidrofóbicos, lo que reduce la sensibilidad al agua y mejora las propiedades mecánicas y de barrera en los materiales TPS (Gáspár et al., 2005; Seidenstücker y Fritz, 1999; Wesslén y Wesslén, 2002).

El esfuerzo de tensión dado en TPS por el uso de fibras de diferentes orígenes hasta de un $30 \%$, fue investigado, demostrándose que la fibra reduce el envejecimiento observado en el material y retarda los procesos de retrogradación dentro de la matriz termoplástico y mejora las propiedades mecánicas y de barrera (Bodros et al., 2007; Ma et al., 2005; Peressini et al., 2003; Seidenstücker y Fritz, 1999).

\section{Envejecimiento y biodegradación}

En esta categoría se reportan estudios que muestran una teoría base para la observación del envejecimiento presentado en diferentes TPS y el cambio en el peso molecular, utilizando diferentes métodos tales como el de Tool-Naraynaswamy (Serghat et al., 1999), difracción de Rayos-X (Forssell et al., 1998), calorimetría de barrido diferencial (DSC) (Forssell et al., 1998; Lim et al., 2001; Mali et al., 2005b; Tan et al., 2004), microscopía de fuerza atómica (AFM) (Kuutti et al., 2001; Thiré et al., 2003a; Thiré et al., 2003b), microscopía de barrido diferencial (SEM) (Acosta et al., 2006a; García et al., 2000; Mali et al., 2002; Mali et al., 2004), microscopia óptica de alta resolución (MOAR) (Acosta et al., 2006b; Fishman et al., 2004); cromatografía de permeación por gel (GPC) (Myllyäki et al., 1997), resonancia magnética nuclear (NMR) y espectroscopia infrarroja por transformada de Fourier (FT-IR) (Smits et al., 1998). La movilidad del agua en estos sistemas de termoplastificación fue monitoreada usando estudios de sorción y difusión durante el almacenamiento (Frigant et al., 1998; Mali et al., 2005a; Tsiapouris y Linke, 2002). Además, se llevaron a cabo estudios de biodegradación usando enzimas que degradan rápidamente el TPS por la $\alpha$-amilasa. Se concluyó que, la amilosa fue parcialmente resistente al ataque de la $\alpha$-amilasa dada la presencia de almidón residual en el material degradado y que necesario continuar las investigaciones con otro tipo de enzimas que posiblemente aceleren el proceso de biodegradación (Araújo et al., 2004; Sitohy y Ramadan, 2001). Además, se evaluó el tiempo de biodegradación en diferentes mezclas de TPS+biopolímeros y TPS+plastificantes. Se encontró que en materiales de TPSs+plastificantes su biodegradación es mas rápida que en TPSs+biopolímeros (Demirgöz et al., 2000; Jayasekara et al., 2002; Preechawong et al., 2005; Petnamsin et al., 2000; Reis et al., 1997).

\section{Mezclas para el desarrollo de nuevos materiales}

En esta categoría se incluyen investigaciones que utilizan el TPS en mezclas con otros materiales para aplicaciones médicas y no alimentarías. Mezclas de TPS+PLA (acido poliláctico) o TPS+PCL (policaprolactana) ayudan a promover la resistencia a la absorción de agua del TPS y mejoran las propiedades mecánicas en los productos expandidos usados como amortiguadores biodegradables que remplazan los productos sintéticos (Cha et al., 2001; Mihai et al., 2007; Preechawong et al., 2005; Petnamsin et al., 2000; Qi y Milford, 2001; Wang et al., 2007; Xu et al., 2005). Igualmente, mezclas de TPS+nanoarcilla le mejoran el comportamiento en las propiedades mecánicas, térmicas y de barrera (Chen y Evans, 2005; Chiou et al., 2005; Dean et al., 2007; Huang et al., 2006). Comportamiento similar fue el mostrado y evaluado en matrices TPS+arcilla (Wilhelm et al., 2003). Mezclas de TPS+quitósan han mostrado disminución en los diferentes procesos de retrogradación ocurridos durante el almacenamiento, dada la reorientación de las moléculas de amilosa y amilopectina dentro de la matriz termoplástica, además la transmisión de vapor de agua disminuye sustancialmente (Bangyekan et al., 2006). Otro material ideal es el TPS+hidroxiapatita, biomaterial usado en implantes compatibles con el sistema óseo en humanos (Leonora et al., 2003). 


\section{CONCLUSION}

La revisión de los artículos publicados permite entender el comportamiento de los almidones cuando son termoplastificados y visualizar los vacíos en donde es necesario enfocar futuras investigaciones que podrán conducir al desarrollo de nuevos productos al servicio de la humanidad. Sin embargo el principal reto se encuentra en disminuir la sensibilidad a la humedad y en retardar los procesos de retrogradación en este tipo de materiales, lo cual disminuirá las limitaciones en el uso de estos precursores en diversas aplicaciones. Por ello, se requieren más estudios sobre mezclas de almidón+plastificantes en el desarrollo de TPS que se enfoquen en disminuir la absorción de agua y retardar los diferentes procesos de recristalización del material para evitar la fragilidad o rigidez desarrollada durante el almacenamiento.

\section{REFERENCIAS}

Acosta, $\mathrm{H}$; Processing and physical-chemical characterization of steam flaked sorghum hybrids. dissertation (Doctor on philosophy). Texas A\&M University. Technology Faculty, Food Science and Technology. Texas, USA (1997).

Acosta, H. y otros seis autores; Efecto de las variables de operación de un extrusor de uso sencillo sobre las propiedades mecánicas de almidón termoplástico hecho de almidón agrio de yuca, Rev. Biotecnol. Sector Agropecuario y Agroindustrial, ISNN: 1692-3561 (en línea), 2 (1), 18-26 (2004). http://www.unicauca.edu.co/biotecnologia/ediciones/vol2/Art22.pdf. Acceso: 20 de junio (2007).

Acosta, H. y otros tres autores; Morfología superficial de almidones termoplásticos agrio de yuca y nativo de papa observados por microscopía óptica y microscopía de fuerza atómica, Rev. Inf. Tecnol, ISSN: $0718-0764$ (en línea), 17 (3), 63-70 (2006a). http://www.scielo.cl/scielo.php?script=sciarttext\&pid=S0718-07642006000300010\&lng=es\&nrm=iso-\&tlng=es. Acceso: 15 de junio (2007).

Acosta, H., H.S. Villada y P.A. Prieto; Envejecimiento de almidones termoplásticos agrios de yuca y nativos de papa por Microscopía de fuerza atómica, Rev. Inf. Tecnol, ISSN: 0718-0764 (en línea) 17 (3), 71-78 (2006b), http://www.scielo.cl/scielo.php?script=sciarttext\&pid=S0718-07642006000300011\&lng=es\&nrm=iso\&tlng=es. Acceso: 15 de Enero (2007).

Aichholzer, W. y H.G. Fritz; Rheological characterization of thermoplastic starch materials, Starch/Stärke: 50 (2-3), 77-83 (1998).

Araújo, M.A., A.M. Cunhac. y M. Mota; Enzymatic degradation of starch-based thermoplastic compounds used in protheses, Biomaterials: 25 (13), 2687-2693 (2004).

Avérous, L. y C. Frigant; Association between plasticized starch and polyesters: processing and perfomances of injected biodegradable systems, Polym. Eng. Sci: 41 (5), 727-734 (2001).

Avérous, L. y otros tres autores; Properties of biodegradable multilayer films based on plasticized wheat starch, Starch/Stärke: 53 (8), 372-380 (2001).

Avérous, L. y otros tres autores; Starch-based biodegradable materials suitable for thermoforming packaging, Starch/Stärke: 53 (8), 368-371 (2003).

Avérous, L. y N. Boquillon; Biocomposites based on plasticized starch: thermal and mechanical behaviours, Carbohydr. Polym: 56 (2), 111-122 (2004).

Baldwin, P.; Studies on the surface chemistry, minor component composition and structure of granule starches. Thesis (Doctor on philosophy). The University of Nottingham. Uk (1995)

Bangyekan, C., D. Aht-Ong. y K. Srikulkit; Preparation and properties evaluation of chitosan-coated cassava starch films, Carbohydr. Polym: 63 (1), 61-71 (2006). 
Bastioli, C; Global status- production of biobased packaging, Starch/Stärke: 53 (8), 351-355 (2001).

Bello, P. y otros tres autores; Isolation and partial characterization of amaranth and banana starches, Starch/Stärke: 50 (10), 409-413 (1998).

Bernal, M.L. y otros siete autores; Variación de los tiempos de fermentación de almidón agrio y de la velocidad de rotación del tornillo de un extrusor de uso sencillo en la obtención de almidón TPS. Rev. Biotecnol. Sector Agropecuario y Agroindustrial, ISNN: 1692-3561 (en línea), 3 (1), 8-13 (2005). http://www.unicauca.edu.co/biotecnologia/ediciones/vol3/Art31.pdf. Acceso: 20 de junio (2007).

BiologicaL Materials For Non-Food Products; Thermoplastic starches for industrial non-food uses (1998), http://www.biomatnet.orgsecureAirF234.htm. Acceso: 8 de junio (2007).

Bindzus, W. y otros tres autores; Application of an in-line viscometer to determine the shear stress of plasticizer wheat starch, Starch/Stärke: 54 (6), 243-251 (2002a).

Bindzus, W. y otros cinco autores; Glass transition of extruder wheat, corn and rice starch, Starch/Stärke: 54 (9), 393-400 (2002b).

Blacido, D.T., P.J. Sobral. y F.C. Menegalli; Development and characterization of biofilms based on Amaranth flour (Amaranthus caudatus), J. Food Sci: 67 (3), 215-223 (2005).

Bodros, E. y otros tres autores; Could biopolymers reinforced by randomly scattered flax fibre be used in structural applications?, Comp. Sci. Technol.: 67 (3-4), 462-470 (2007).

Brown, S.B. y C.M. Orlando; Reactive extrusion, En: Encyclopedia of Polymer Science and Engineering, Ed. Wiley, Vol. 14, pp. 169-170, New York, USA (1988).

Cha, J.Y. y otros cuatro autores; Physical properties of starch-based foams as affected by extrusion temperature and moisture content, Ind. Crops and Product: 14 (1), 23-30 (2001).

Chen, B. y J.R.G. Evans; Thermoplastic nanocomposites, Carbohydr. Polym: 61 (4), 455-463 (2005).

Chiou, B.S. y otros tres autores; Rheology of starch-clay nanocomposites, Carbohydr. Polym: 59 (4), 467-475 (2005).

Curvelo, A.A.S., A.J.F. Carvalho. y J.A.M. Agnelli; Thermoplastic starch-cellulosic fibers composites: preliminary results, Carbohydr. Polym: 45 (2), 183-188 (2001).

De Graaf, A.R., A.P. Karman. y L.P. Janssen; Material properties and glass transition temperature of different thermoplastic starches after extrusion process, Starch/Stärke: 55 (2), 80-86 (2003).

Dean, K., L. Yu. y D.Y. Wu; Preparation and characterization of melt-extruded thermoplastic starch/clay nanocomposites, Comp. Sci. Technol: 67 (3-4), 413-421(2007).

Demiate, I.N. y otros cuatro autores; Relationship between baking behaviour of modified cassava starches and determined by FT-IR spectroscopy, Carbohydr. Polym: 42 (2), 149-158 (2002).

Demirgöz, D. y otros cinco autores; Chemical modification of starch based biodegradable polymeric blends: effects on water uptake, degradation behaviour, Polym. Degrad. Stab: 70 (2), 161-170 (2000).

Dole, P. y otros cuatro autores; Gas transport properties of starch based films, Carbohydr. Polym: 58 (3), 335-343 (2004).

Fama, L. y otros tres autores; Mechanical properties of tapioca-starch edible films containing sorbates, LWT-Food Sci. Technol: 38 (6), 631-639 (2005). 
Fang, J.M. y otros tres autores; An investigation of the use of recovered vegetable oil for the preparation of starch thermoplastics, Carbohydr. Polym: 50 (4), 429-434 (2002).

Finkenstadt, V.L. y J.L. Willett; A directcurrent resistance technique for determining moisture content in native starches and starch-based plasticized materials, Carbohydr. Polym: 55 (2), 149-154 (2004).

Fishman, M.L. y otros tres autores; Extrusion of pectin and glycerol with various combinations of orange albedo and starch, Carbohydr. Polym: 57 (4), 401-413 (2004).

Follain, N. y otros tres autores; Properties of starch based blends. Part 2. Influence of poly vinyl alcohol addition and photocrosslinking on starch based, Carbohydr. Polym: 60 (2), 185-192 (2005).

Forssell, P.M. y otros tres autores; Phase and glass transition behaviour of concentrated barley starch-glycerol-water mixtures, a model for thermoplastic, Carbohydr. Polym: 34 (4), 275-282 (1997)

Forssell, P.M. y otros cuatro autores; Ageing of rubbery thermoplastic starches prepared from barley and oat starches, Carbohydr. Polym: 30 (1), 7-12 (1998).

Forssell, P.M y otros tres autores; Oxygen permeability of amylose and amylopectin film, Carbohydr. Polym: 47 (2), 125-129 (2002).

Frigant, C. y otros cinco autores; A biogradable starch based coating to waterproof hydrophilic materials, Starch/Stärke: 50 (7), 292-296 (1998).

García, M.A., M.N. Martino. y N.E. Zaritzky; Microstructural Characterization of Plasticized StarchBased Film, Starch/Stärke: 52 (4), 118-124 (2000).

Gáspár, M. y otros cuatro autores; Reducing water absorption in compostable starch-based plastics, Polym. Degrad. Stabili: 90 (3), 563-569 (2005).

Gaudin, S. y otros cuatro autores; Plasticized and mobility in starch-sorbitol films, J. Cereal Sci: 29 (3), 273-284 (1999).

Halley, P. y otros nueve autores; Developing biodegradable mulch films from starch-based polymers, Starch/Stärke: 53 (8), 362-367 (2003).

Hoover, R; Composition molecular structure, and physicochemical properties of tuber and root starches: a review, Carbohydr. Polym: 45 (3), 253-267 (2001).

Huang, M., J. Yu. y X. Ma; High mechanical performance MMT-urea and formamide-plasticized thermoplastic cornstarch biodegradable nanocomposites, Carbohydr. Polym: 63 (3), 393-399 (2006).

Jayasekara, R. y otros ocho autores; Biodegradation and ecotoxicity evaluation of a bionolle and starch blend and its degradation products in compost, Int. Biodeter. Biodegr.: 51 (1), 77- 81 (2002).

Jiugao, Y. y otros cinco autores; A study on the properties of starch/glycerine blend, Starch/Stärke: 50 (6), 246-250 (1998).

Kuutti, L. y otros cuatro autores; AFM in estudies of thermoplastic starches during ageing, Carbohydr. Polym: 37 (1), 7-12 (2001).

Kenshi, M. y otros cuatro autores; Thermal and viscoelastic properties of alginate/poly (vinyl alcohol) blends cross-linked with calcium tetraborate, Carbohydr. Polym: 39 (2), 139-144 (1999).

Kuakoon, P. y otros tres autores; A compartive study of edible canna (Canna edulis) starch from different cultivars. Part I. Chemical composition, Carbohydr. Polym: 53 (3), 317-324 (2003). 
Leonora, I.B. y otros cuatro autores; In vitro bioactivity of starch thermoplastic/hydroxyapatite composite biomaterials: in situ study atomic force microscopy, Biomaterials: 24 (4), 579-585 (2003).

Lim, S.T., E.H. Chang. y H.J. Chung; Thermal transition characteristics of heat-moisture treated corn and potato starches, Carbohydr. Polym: 46 (2), 107-115 (2001).

Lindeboom, N., P.R. Chang. y R.T. Tyler; Analytical, biochemical and physicochemical aspects of starch granule size, with emphasis on small granule starches:; Starch/Stärke: 56 (3-4), 89-99 (2004).

Ma. X. y J. Yu; The plasticizers containing amide groups for thermoplastic starch, Carbohydr. Polym: 57 (2), 197-203 (2004).

Ma, X., J. Yu. y J.F. Kennedy; Studies on the properties of natural fibers-reinforced Thermoplastic starch composites, Carbohydr. Polym: 62 (1), 19-24 (2005).

$\mathrm{Ma}, \mathrm{X} . \mathrm{y}$ otros tres autores; The effects of different plasticizers on the properties of thermoplastic starch as solid polymer electrolytes, Macromol. Mater. Eng.: 292 (4), 503-510 (2007).

Mali, S. y otros cuatro autores; Microstructural characterization of yam starch film, Carbohydr. Polym: 50 (4), 379-386 (2002).

Mali, S. y otros cuatro autores; Barrier, mechanical and optical properties of plasticized yam starch film, Carbohydr. Polym: 56 (2), 129-135 (2004).

Mali, S. y otros tres autores; Water sorption and mechanical properties of cassava starch films and their relation to plasticizing effect, Carbohydr. Polym: 60 (3), 283-289 (2005a).

Mali, S. y otros cuatro autores; Mechanical and thermal properties of yam starch films, Food Hydrocolloids: 19 (1), 157-164 (2005b).

Mater Bi; 2007. http://www.promotions.org.uk/biodegradableproduc.htm. Acceso: 14 de Enero (2007).

Mihai, M. y otros tres autores; Extrusion foaming of semi-srystalline PLA and PLA/Thermoplastic starch blends, Macromol. Biosci: 7 (7), 907-920 (2007).

Myllyäki, O. y otros cinco autores; Depolimerization of barley starch during extrusion in water glycerol mixtures. LWT-Food Sci. Technol: 30 (4), 351-358 (1997).

Myllärinen, P. y otros tres autores; The crystallynity of amylose and amylopectin film, Carbohydr. Polym: 48 (1), 41-48 (2002a).

Myllärinen, P. y otros tres autores; Effect of glycerol on behaviour of amylose and amylopectin films, Carbohydr. Polym: 50 (4), 355-361(2002b).

Nashed, G., P.G.R. Rutgers. y P.A. Sopade; The plasticization effect of poliol and water on the gelatinization of wheat starch, Starch/Stärke: 55 (3-4), 131-137 (2003).

Novamont. TPS for Industrial Non-Food Uses; 2007. http://www.biomatnet.org/secure/Other/S1281. htm. Acceso: 14 de Enero (2007).

Onteniente, J.P. y otros tres autores; Fully biodegradable lubricated thermoplastic wheat starch: Mechanical and rheological properties of an injection grade, Starch/Stärke: 52 (4), 112-117 (2000).

Orts, J. W. y otros cinco autores; Blends of starch with ethylene vinyl alcohol copolymers: effect of water, glycerol, and amino acids as plasticizers, Polym. Adv. Technol: 18 (8), 629-635 (2007). 
Pareta, R. y M.J. Edirisinghe; A novel method for the preparation of starch films and coatings, Carbohydr. Polym: 63 (3), 425-431 (2006).

Parra, D.F. y otros tres autores; Mechanical properties and water transmission in some blends of cassava starch edible films, Carbohydr. Polym: 58 (4), 475-481 (2004).

Peressini, D. y otros cuatro autores; Starch-methylcellulose based edible films: rheological properties of film-forming dispersions, J. Food Eng: 59 (1), 25-32 (2003).

Petersson, M. y M. Standing; Water vapour permeability and mechanical properties of mixed starchmonoglyceride films and effect of film forming conditions, Food Hydrocolloids: 19 (1), 123-132 (2005).

Petnamsin, C., N. Termvejsayanon. y K. Sriroth; Effect of particle size on physical properties and biodegradability of cassava starch/polymer blend, J. Nat. Sci.: 34 (2), 254-261(2000).

Philippe, D; Seminario textura y reología de alimentos. Memorias, Dpto. Eng. Alimentos, Universidad del Valle, Cali-Colombia (1995).

Preechawong, D. y otros tres autores; Preparation and characterization of starch/poly (L-lactic acid) hybrid foams, Carbohydr. Polym: 59 (3), 329-337 (2005).

Qi, F. y A.H. Milford; Characteristics of biodegradable Materbi-starch based foams as affected by ingredient formulations, Ind. Crops and Prod: 13 (3), 219-227 (2001).

Reis, R.L. y otros tres autores; Processing and in vitro degradation of Starch/EVOH thermoplastic blends, Polym. Int: 43 (2), 347-352 (1997).

Rhim, J.W.; Physical and mechanical properties of water resistant sodium alginate films, LWT-Food Sci. Technol: 37 (3), 323-330 (2004).

Seidenstücker, T. y H.G. Fritz; Compounding procedure, processing behavior and property profiles of polymeric blends on thermoplastic poly (ester-urethanes), Starch/Stärke: 51 (2-3),93-102 (1999).

Serghat, D.H., A.G. Copinet. y Y. Couturier; Aerobic biodegradation of extruded polymer blends with native starch as major component, Starch/Stärke: 51 (10), 369-375 (1999).

Shi, R. y otros seis autores; Characterization of citric acid/glycerol co-plasticized thermoplastic starch prepared by melt blending, Carbohydr. Polym: 69 (4), 748-755 (2007).

Shogren, R.L., G.F. Fanta. y W.N. Doane; Development of starch based plastics, a reexamination of selected polymer systems in historical prospective, Starch/Stärke: 45 (8), 276-280 (1993).

Sitohy, M.Z. y M.F. Ramadan, Degradability of different phosphorylated starches and thermoplastic films prepared from corn starch phosphomonoesters, Starch/Stärke: 53 (7), 317-322 (2001).

Smits, A.L.M. y otros cuatro autores; Ageing of starch based systems as observed with FT-IR and solid state NMR spectroscopy, Starch/Stärke: 50 (11-12), 478-483 (1998).

Smits, A.L.M. y otros tres autores; The influence of various small plasticisers and maltooligosaccharides on the retrogradation of (partly) starch, Carbohydr. Polym: 51 (4), 417-424 (2003).

Stepto, R.F.T; Understanding of thermoplastic starch, Macromol. Symp: 245(1), 571-577 (2006).

Tan, I. W. y otros tres autores; Estimating the specific heat capacity of starch-water-glycerol systems as a function of temperature and compositions, Starch/Stärke: 56 (1), 6-12 (2004). 
Thiré, M.S.M.R., A.R. Simâo. y T.C. Andrade; Hihg resolution imaging of the microstructure of maize starch films, Carbohydr. Polym: 54 (2), 149-158 (2003a).

Thiré, M.S.M.R., R.A. Simâo. y Andrade, T.C; Investigation of the surface morphology of plasticized cornstarch films, Acta Micro.: 12 (1), 175-179 (2003b).

Tsiapouris, A. y L. Linke; Water vapour sorption determination of starch based porous packaging materials, Starch/Stärke: 52 (2-3), 53-57 (2002).

Van soest, J.J.G. y otros tres autores; Bioplastic, Ind. Crops and Prod: 5(1), 11-22 (1996a).

Van Soest, J.J.G. y otros tres autores; Changes in the mechanical properties of thermoplastic potato starch in relation with changes in B-type crystallinity, Carbohydr. Polym: 29 (3), 225-232 (1996b).

Van Soest, J.J.G. y otros tres autores; Influence of glycerol on the melting of potato starch, Ind. Crops and Prod: 5 (1), I-9 (1996c).

Van Soest, J.J.G. y P. Essers; Influence of amylose/amylopectin ratio on properties of extruded starch plastic sheets, J. Macromol. Sci., Part A: 34 (9), 1665-1689 (1997).

Van Soest, J.J.G y J.F.G. Vliegenthart; Crystallinity in starch plastics: consequences for material properties, Trends in Biotechnology: 15 (6), 208-213 (1997).

Wang, N. y otros tres autores; Influence of formamide and water on the properties of thermoplastic starch/poly (lactic acid) blends, Carbohydr. Polym: Article in Press. (2007).

Werner, W. y E. Strobel; Compounding of thermoplastic starch with twin-screw extruders, Starch/Stärke: 43 (4), 138-145 (1991).

Wesslén, K.B. y B. Wesslén; Synthesis of amphiphilic amylase and starch derivatives, Carbohydr. Polym: 47 (4), 303-311 (2002).

Wilhelm, H.M. y otros tres autores; Starch films- mineral, Carbohydr. Polym: 52(2), 101-110 (2003).

Wilpiszewska, K. y T. Spychaj, Chemical modification of starch with hexamethylene diisocyanate derivatives, Carbohydr. Polym: Article in Press. (2007).

Xie, F. y otros tres autores; Starch using reactive extrusion, Starch/Stärke: 58 (3-4), 131-139 (2006).

Xu, Y.X., Y. Dzenis. y M.A. Hanna; Water solubility, thermal characteristics and biodegradability of extruded starch acetate foams, Ind. Crops and Prod: 21 (3), 361-368 (2005).

Yang, J., J. Yu. y X. Ma; Study on the properties of ethylenebisformamide and sorbitol plasticized corn starch (ESPTPS), Carbohydr. Polym: 66 (1), 110-116 (2006).

Yuryev, V.P., I.E. Nemirosvkaya. y T.D. Maslova; Phase state of starch gels at differrent water contents, Carbohyd. Polym: 26 (1), 43-46 (1995).

Zhai, M., F. Yoshii. y T. Kumeb; Radiation of starch-based, Carbohydr. Polym: 52(3), 311-317 (2003).

Zhang, Y. y J.H. Han; Mechanical and thermal characteristics of pea starch films plasticized with monosaccharides and polyols, J. Food Sci: 71 (2), 109-118 (2006).

Zhang, S.D. y otros cinco autores; Modified corn starches with improved comprehensive properties for preparing thermoplastics, Starch-Stärke: 59 (6), 258-268 (2007). 